\title{
Ketamine attenuates osteoarthritis of the knee via modulation of inflammatory responses in a rabbit model
}

\author{
WEI LU ${ }^{1}$, LIN WANG ${ }^{1}$, CHUNXIN WO $^{1,2}$ and JING YAO ${ }^{1}$ \\ ${ }^{1}$ Department of Anesthesiology, Guizhou Medical University; ${ }^{2}$ Division of Pain Management, \\ The Affiliated Hospital of Guizhou Medical University, Guiyang, Guizhou 550004, P.R. China
}

Received May 28, 2015; Accepted March 31, 2016

DOI: $10.3892 / \mathrm{mmr} .2016 .5164$

\begin{abstract}
The aim of the present study was to investigate the efficacy of ketamine in attenuating osteoarthritis (OA) and modulating the expression of inflammatory mediators. A rabbit OA model was established by knee immobilization using plaster bandages. After six weeks, rabbits were randomly allocated into four groups ( $\mathrm{n}=6 /$ group): Normal saline, $\mathrm{Ket}_{60}$, $\mathrm{Ket}_{100}$, and $\mathrm{Ket}_{200}$ and twice a week for four weeks the rabbits received an intra-articular injection of saline, or 60, 100 or $200 \mu \mathrm{mol} / 1$ ketamine, respectively. One week after the final injection, samples of synovial membrane, synovial fluid and articular cartilage were isolated. The pathological changes were assessed by general observation, hematoxylin and eosin staining and Alcian blue/periodic-acid Schiff staining. Cartilage pathology was assessed using Mankin's scoring system. Tumor necrosis factor (TNF)- $\alpha$ and interleukin (IL)-10 levels in the synovial fluid were measured by enzyme-linked immunosorbent assays. The nuclear factor (NF)- $\kappa \mathrm{B}$ p65 subunit expression level in cartilage samples was determined by immunohistochemistry. OA was characterized by morphological changes in the articular surface, cartilage lesions, infiltration of inflammatory cells and a significantly increased Mankin's score. Elevated TNF- $\alpha$ and reduced IL-10 levels in the synovial fluid, along with increased p65 expression levels in the cartilage were observed in OA rabbits. Intra-articular injection of ketamine ameliorated the pathological characteristics of OA, reduced the Mankin's score, decreased TNF- $\alpha$ and NF- $\kappa$ B p65 expression levels, and increased the level of IL-10 expression in a dose-dependent manner. Thus is was demonstrated that Ketamine suppresses the inflammatory response in OA by modulating inflammatory mediator expression levels in a rabbit model of OA.
\end{abstract}

Correspondence to: Ms. Chunxin Wo, Department of Anesthesiology, Guizhou Medical University, 4 Beijing Road, Guiyang, Guizhou 550004, P.R. China

E-mail: adjqy@126.com

Key words: ketamine, osteoarthritis, tumor necrosis factor- $\alpha$, interleukin-10, nuclear factor- $\kappa \mathrm{B}$

\section{Introduction}

Osteoarthritis $(\mathrm{OA})$ is the most common form of progressive joint disease in the elderly, which has a reported prevalence of $34.18 \%$ in women and $30.18 \%$ in men, aged 65 years or older, in the United States of America (1). A previous study conducted in Beijing, China, revealed a higher prevalence of OA in Chinese women of a similar age (46.16\%) and a comparable prevalence in Chinese men (27.16\%) (2). OA is associated with degenerative changes in the joints, along with joint diseases caused by loss of the cartilage matrix (3). Furthermore, previous studies have highlighted the role of inflammation in the pathogenesis of OA $(4,5)$. Elevated expression levels of pro-inflammatory cytokines and mediators, including interleukin (IL)-1, IL-6, tumor necrosis factor (TNF)- $\alpha$ and nitric oxide (NO) are present in the serum and synovial fluid of OA patients $(6,7)$. Anti-inflammatory agents, such as IL- $1 \beta$ inhibitor, diacerein and TNF- $\alpha$ inhibitors, infliximab and etanercept have demonstrated promising clinical efficacy for the treatment of OA (8-10). However, to the best of our knowledge, there have been no reports of therapeutic strategies that could simultaneously engage multiple inflammatory mediators and alleviate inflammation-induced tissue damage.

Recent studies have extended the functional scope of glutamate beyond neuronal tissues to a wide range of tissue and cell types, exhibiting an autocrine or paracrine effect (11-13). McNearney et al (14) revealed increased levels of glutamate, and pro-inflammatory cytokines and chemokines in the synovial fluid of patients with active arthritis. Glutamate interacts specifically with the N-methyl-D-aspartate (NMDA) receptor and activates the downstream signaling cascade (15). Flood et al (16) proposed the role of NMDA receptor in inflammatory joint damage in rheumatoid arthritis. In an animal study, injecting glutamate into the knee joint of rats led to thermal hyperalgesia and mechanical allodynia, which was alleviated by subsequent injections of NMDA receptor antagonists (17). Magnesium is an NMDA receptor antagonist, and an articular injection has been shown to improve the degree of pain following arthroscopic surgery and improve experimental rat bone arthritis $(18,19)$. Results from the above-mentioned studies indicate the therapeutic potential of glutamate/NMDA receptor signaling in OA. Ketamine is a non-competitive antagonist of the NMDA receptor, and has been widely administered as an anesthetic and pain killer $(20,21)$. Recent studies 
indicate that ketamine exhibited potent anti-inflammatory activity in various inflammatory disease models when administered at or below clinical dosages. Ketamine antagonizes the NMDA receptor to regulate calcium influx, increases the intracellular concentration of cyclic adenosine monophosphate, inhibits the formation of oxygen radicals and inducible NO synthase (iNOS) following polymorphonuclear activation, and modulates the production of various pro-inflammatory mediators (21). Mechanistic studies indicate that ketamine directly inhibits the expression of nuclear factor (NF)- $\mathrm{kB}$, which is a master regulator of pro-inflammatory cytokine transcriptions (22). In addition, ketamine exhibits protective effects against oxidative stress by inhibiting iNOS activity and decreasing nitrite/nitrate levels (23). These findings highlight the therapeutic potential of ketamine in inflammatory disorders, including OA.

In the present study, a rabbit OA model was established by immobilizing the knee joint, as previously described (24), and the efficacy of different doses of ketamine in ameliorating the inflammatory response was evaluated. In addition, the current study investigated the anti-inflammatory role of ketamine in regulating multiple inflammatory cytokines and signaling molecules for possible therapeutic application in OA.

\section{Materials and methods}

Animals and reagents. Thirty skeletally mature, male, New Zealand white rabbits (weight, $2.5-3 \mathrm{~kg}$ ) were obtained from the Experimental Animal Center of Guiyang Medical School (Guiyang, China) and were acclimated for one week prior to the experimental procedures. All animal procedures were approved by the Institutional Ethics Committee.

The ketamine used in the present study was a 1:1 racemic mixture of two enantiomers, which was purchased from Yichang Humanwell Pharmaceutical Co.,Ltd. (Yichang, China). Enzyme-linked immunosorbent assay (ELISA) kits for IL-10 and TNF- $\alpha$, and the anti-NF- $\mathrm{BB}$ p65 antibody were purchased from Shanghai Bogoo Biotechnology Co., Ltd. (Shanghai, China). The 3,3'-diaminobenzidine (DAB) Substrate kit was obtained from ZSGB-Bio Co., Ltd. (Beijing, China), and Alcian blue/periodic acid-Schiff (AB/PAS) Stain kit was obtained from Huanyu Jinying Technology Co., Ltd. (Beijing, China).

Rabbit OA model and ketamine treatment. Twenty-four rabbits were randomly selected to establish the OA model. The left knee joint was immobilized $(3 \mathrm{~cm}$ above the rear ankle to $1.5 \mathrm{~cm}$ below the groin) for six weeks using plaster bandages, with the knee flexed at $30-40^{\circ}$. Dorsalis pedis pulses were detected on each side. Plaster tightness was examined for three consecutive days after creating the model and were adjusted as appropriate. The rabbits were housed in separate cages and forced to move or exercise occasionally. Six rabbits were left untreated and served as a normal control.

After six weeks, the plaster was removed and rabbits were randomly allocated into four groups: Normal saline, $\operatorname{Ket}_{60}$, $\mathrm{Ket}_{100}$ and $\mathrm{Ket}_{200}$. Saline or various concentrations of ketamine $(60,100$ and $200 \mu \mathrm{mol} / \mathrm{l})$ were injected into the articular cavity in $0.5-\mathrm{ml}$ volumes, twice a week for four weeks. One week after the last injection, sample collection was conducted, then all rabbits were sacrificed under intravenous anesthesia with
$25 \%$ urethane $(4 \mathrm{ml} / \mathrm{kg}$; Shanghai-Rui Biological Technology Co., Ltd., Shanghai, China) by air injection $(20 \mathrm{ml})$ into an ear marginal vein.

Sample collection. The thigh was rotated and an incision was made on the inner side in order to peel back the skin. The quadriceps were dissected $0.5 \mathrm{~cm}$ above the knee joint, and separated from the femur and patella. The synovial membrane under the patella was removed using ophthalmic scissors (Shanghai Yuyan Instruments Co., Ltd., Shanghai, China) and rinsed in ice-cold phosphate-buffered saline solution (Shanghai-Rui Biological Technology Co., Ltd.), and fixed in $10 \%$ neutral buffered formalin (BioSynTech, Beijing, China) for $24 \mathrm{~h}$ followed by dehydration, clearing, wax infiltration and embedding in paraffin (Junruishengwu Technology Corporation, Shanghai, China) for further histological examination. The samples were $0.5 \times 1 \times 0.5 \mathrm{~cm}$ in size.

The femoral condyle and a portion of the cartilage from the knee joint were removed, rinsed and immediately fixed in $10 \%$ neutral buffered formalin for $24 \mathrm{~h}$. Samples were decalcified prior to histological examination.

Synovial fluid was obtained from the knee joint by injecting $0.5 \mathrm{ml}$ saline solution followed by aspiration, which was performed three times. Synovial fluid $(\sim 0.8-1 \mathrm{ml})$ was extracted and samples were centrifuged at $2147 \mathrm{x}$ g for $10 \mathrm{~min}$. The supernatants were obtained and stored at $-70^{\circ} \mathrm{C}$ until further use.

General and histopathological examination. The knee joint was observed for signs of synovitis, including joint effusion and swelling. Pathological changes in the articular surface of the medial femoral condyle were evaluated under an Olympus CX41 biological microscope (Olympus Corporation, Tokyo, Japan). Samples were scored according to the following criteria (25): 0, Smooth articular surface with normal color; i) rough articular surface with gray color and small cracks; ii) eroded articular surface with cartilage lesions penetrating the middle layer; iii) ulcers on articular surface with cartilage lesions penetrating the deep layer; and iv) complete loss of cartilage with exposed subchondral bone. Paraffin-embedded tissue samples were sectioned into 3-5- $\mu \mathrm{m}$ thick slices using a DQP-9010 rotary slicer (Shanghai Huayan Equipment Co., Ltd., Shanghai, China). The synovial membrane was stained with hematoxylin and eosin (H\&E; Beijing Solarbio Science \& Technology Co., Ltd., Beijing, China), and mounted with neutral balata (Shanghai-Rui Biological Technology Co., Ltd.); the pathological changes were evaluated by two independent observers. The cartilage was stained using $\mathrm{H} \& \mathrm{E}$ and $\mathrm{AB} / \mathrm{PAS}$, and mounted with neutral balata. Samples were scored by two independent observers according to Mankin's score system (26) and the mean was taken as the final score.

ELISA of IL-10 and TNF- $\alpha$ in the synovial fluid. IL-10 and TNF- $\alpha$ concentration levels in the synovial fluid were measured using specific ELISA kits according to the manufacturer's instructions. Absorbance (optical density value) at a wavelength of $450 \mathrm{~nm}$ was determined using a UV-2600 microplate reader (Shimadzu Corporation, Kyoto, Japan).

Immunohistochemical analysis of $N F-\kappa B$. Immunohistochemical expression of NF- $\mathrm{\kappa B}$ in the cartilage 
was determined using the Labeled Streptavidin Biotin method. Samples were evaluated based on positive DAB staining in the cytoplasm and nucleus of chondrocytes: Weak positive, pale yellow stain; positive, yellow stain; medium positive, brown stain; strong positive, tan stain. Eight fields (magnification, $\mathrm{x} 400$ ) from each section were randomly selected and the positive rate (\%) was determined by the percentage of positively-stained cells. Results were assessed by an investigator and two experienced technicians.

Statistical analysis. Data were expressed as arithmetic or geometric mean \pm standard deviation. Means of the two groups were compared using Levene's test of homogeneity of variance. Homogeneous variance was analyzed by one-way analysis of variance, and pairwise comparison between groups was made by Fisher's least significant difference test. Dunnett's T3 test was performed when there was lack of homogeneity of variances. Positive rates were compared by $\chi^{2}$-test. $\mathrm{P}<0.05$ was considered to indicate a statistically significant difference.

\section{Results}

Evaluation of the rabbit $O A$ model. A rabbit OA model was established by knee immobilization, as described in previous studies $(24,27)$. Following six weeks of immobilization, varying degrees of thigh muscle atrophy and knee stiffness were observed with the loss of the majority of active and passive ranges of motion. Typical OA pathological changes were identified, including hyperemia, swelling and edema in the joint capsule and synovial tissue, as well as joint effusion, rough and dull articular surfaces and cartilage lesions; indicating the successful establishment of the OA model.

Ketamine attenuates $O A$. Various doses (60, 100 and $200 \mu \mathrm{mol} / \mathrm{l})$ of ketamine were intra-articularly injected into rabbits in the $\mathrm{Ket}_{60}, \mathrm{Ket}_{100}$ and $\mathrm{Ket}_{200}$ groups following immobilization. After four weeks of treatment, rabbits in the normal saline group demonstrated the highest gross scores (3.67 \pm 0.52$)$; exhibiting prominent joint effusion and swelling, eroded articular surfaces and cartilage lesions. Rabbits in the Ket $_{60}$ group had marginally lower gross scores (3.17 \pm 0.75$)$; while rabbits in the $\mathrm{Ket}_{100}$ and $\mathrm{Ket}_{200}$ groups exhibited the lowest gross scores $(2.0 \pm 0.00$ and $1.33 \pm 0.82$, respectively), and showed mild joint swelling and a small degree of effusion (Fig. 1).

Synovial membrane samples from all four groups were stained with $\mathrm{H} \& \mathrm{E}$ and the histopathological changes were evaluated. Pronounced inflammatory cell infiltration was observed in the synovial membrane samples from the normal saline group (Fig. 2A). The number of infiltrating inflammatory cells decreased with the increase in ketamine dosage, indicating the attenuation of inflammation (Fig. 2B-D).

$\mathrm{H} \& \mathrm{E}$ staining revealed that the structure and organization of chondrocytes were markedly disrupted and partially replaced by proliferating tissues in the normal saline group (Fig. 3A). Despite obvious chondrocyte proliferation in the $\mathrm{Ket}_{60}$ group, the chondrocyte structure was highly visible with featuring tidemarks (Fig. 3B). Morphology of the articular cartilage was further improved in the $\mathrm{Ket}_{100}$ group, as demonstrated by the normal layer structure observed (Fig. 3C). In

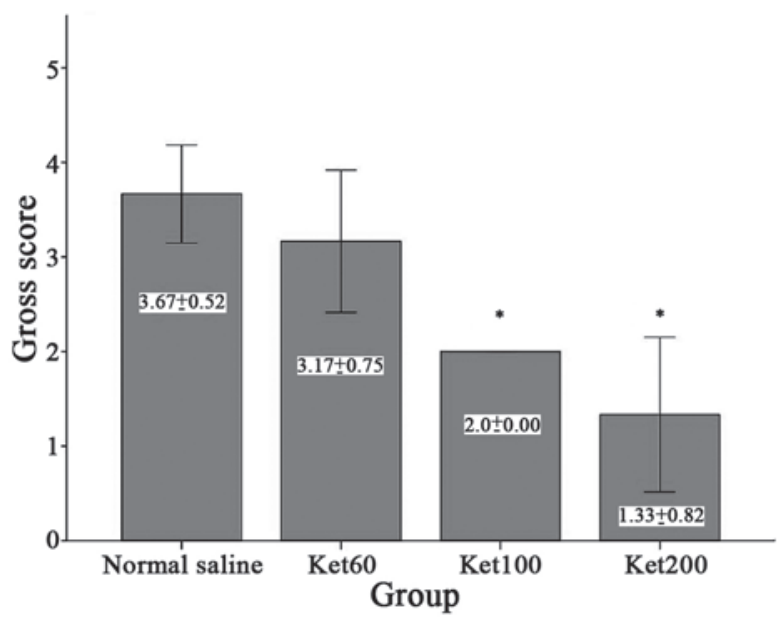

Figure 1. Articular cartilage gross scores in osteoarthritis with or without ketamine. Osteoarthritis rabbits were treated with normal saline or various concentrations of ketamine $(60,100$ and $200 \mu \mathrm{mol} / \mathrm{l})$ twice a week for four weeks. The morphology of the articular surface and cartilage was evaluated, and gross scores for each group were determined. ${ }^{*} \mathrm{P}<0.05$ vs. normal saline group.

the $\mathrm{Ket}_{200}$ group, only mild deformation and disorganization were observed in chondrocytes of the upper layer without any changes in the middle or lower layers (Fig. 3D).

$\mathrm{AB} / \mathrm{PAS}$ staining was performed to indicate cartilaginous tissues. As shown in Fig. 4, the extent of blue staining of cartilaginous tissue samples was significantly reduced in the normal saline group, but revealed a dose-dependent increase following treatment with ketamine. Finally, unlike the normal control group without knee immobilization, rabbits in the normal saline group exhibited the highest Mankin's scores, which indicated the most severe cartilage lesions (Fig. 5). These scores decreased in the ketamine-treated groups $(6.7 \pm 0.9$, $5.8 \pm 0.6$ and 4.1 \pm 0.7 for $\mathrm{Ket}_{60}, \mathrm{Ket}_{100}$, and $\mathrm{Ket}_{200}$, respectively) in a dose-dependent manner, indicating that ketamine treatment ameliorated the joint damage associated with OA.

Ketamine regulates the expression levels of $I L-10, T N F-\alpha$ and $N F-\kappa B$. ELISA and immunohistochemistry revealed significantly elevated pro-inflammatory cytokine, TNF- $\alpha$ expression levels in the synovial fluid of rabbits with immo-

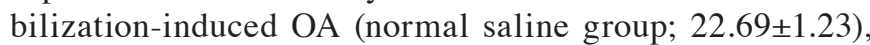
but not in the normal control group (Fig. 6A; $0.00 \pm 0.00$ ). Ketamine treatment significantly decreased TNF- $\alpha$ levels in a dose-dependent manner (from $18.78 \pm 1.51$ to $12.53 \pm 1.34$ in the $\mathrm{Ket}_{60}$ and $\mathrm{Ket}_{200}$ groups, respectively). By contrast, the expression levels of anti-inflammatory cytokine, IL-10 were lower in OA rabbits $(0.38 \pm 0.11)$ when compared with those of the normal controls $(0.49 \pm 0.13)$; however a dose-dependent increase was exhibited in the ketamine-treated groups (Fig. 6B; $0.54 \pm 0.09$ to $0.81 \pm 0.19$ in the $\mathrm{Ket}_{60}$ and $\mathrm{Ket}_{200}$ groups, respectively). The $\mathrm{NF}-\kappa \mathrm{B}$ expression level in the articular cartilage was determined using anti-NF- $\kappa \mathrm{B}$ p65 antibodies. Minimal NF- $\mathrm{B}$ p65 subunit expression levels were detected in chondrocytes of the normal control group, but were markedly elevated in OA rabbits of the normal saline group (Fig. 7A and B). Following intra-articular injection of ketamine, a dose-dependent reduction of NF- $\kappa \mathrm{B}$ p65 expres- 
A

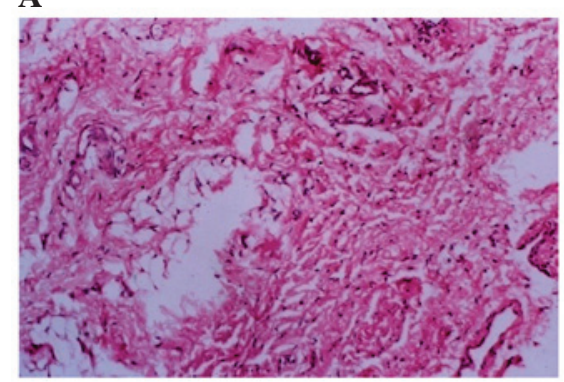

C

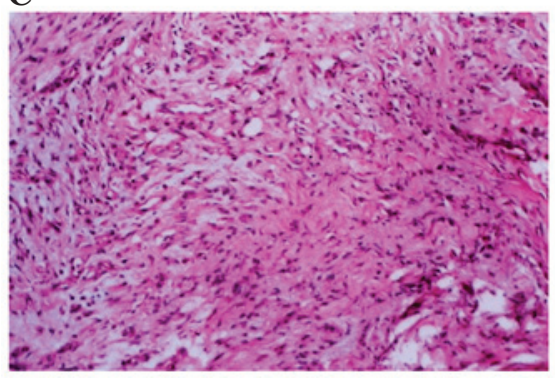

B

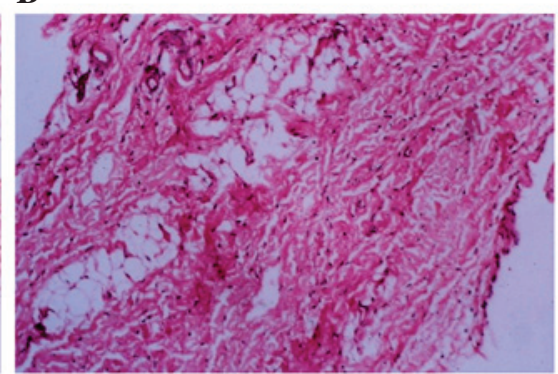

D

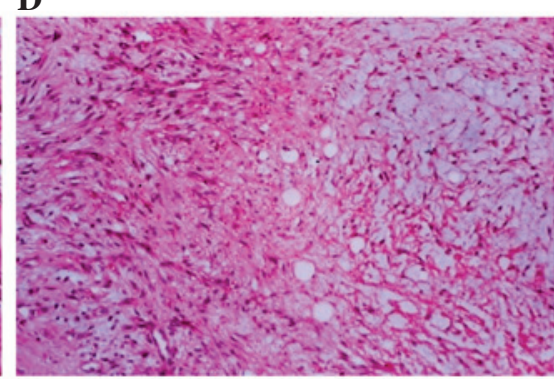

Figure 2. Histopathology of synovial membrane samples from osteoarthritis rabbits with or without 60,100 and $200 \mu$ mol/1 ketamine, cells were stained with hematoxylin and eosin. (A) Normal saline, pronounced inflammatory cells; (B) Ket ${ }_{60}$, a marginal reduction in inflammatory cells; (C) Ket ${ }_{100}$, further reduction in the number of inflammatory cells; and (D) Ket ${ }_{200}$, significantly reduced number of inflammatory cells, indicating attenuated inflammation in the synovial membrane. Magnification, x100.

A

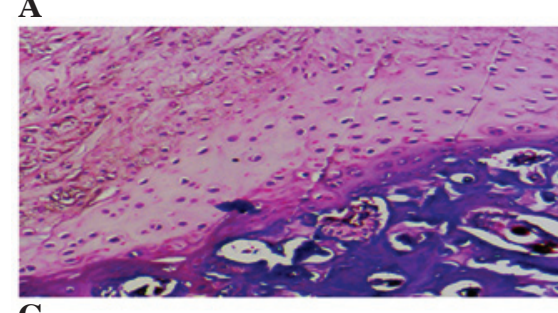

C

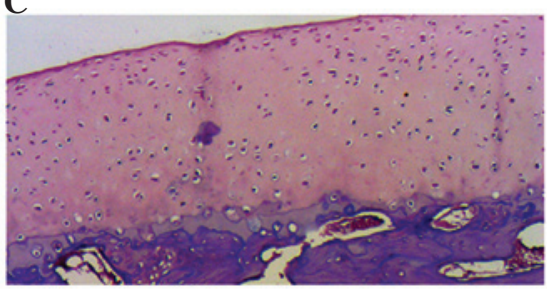

B

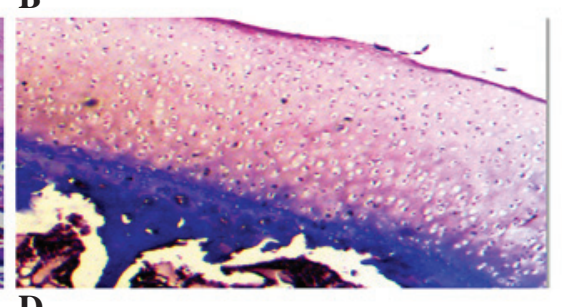

D

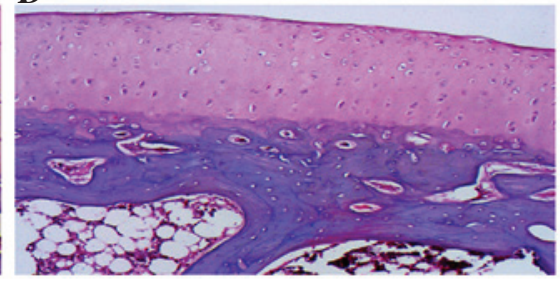

Figure 3. Hematoxylin and eosin staining of articular cartilage samples from osteoarthritis rabbits with or without 60 , 100 and $200 \mu$ mol/1 ketamine: (A) Normal saline, the layer structure of articular cartilage was disrupted and partially replaced by proliferating tissues; (B) Ket ${ }_{60}$, pronounced proliferation of chondrocytes observed, however zonation and tidemarks were visible; (C) $\mathrm{Ket}_{100}$, normal layer structure with tidemarks was observed and a small number of proliferating chondrocytes were observed; and (D) Ket ${ }_{200}$, mild disorganization was identified in the upper layer, however normal structure and chondrocyte organization were observed in the middle and lower layers. Magnification, x200.

sion was observed (Fig. 7C-F; $35.76 \pm 2.69$ to $26.69 \pm 4.68$ in the $\mathrm{Ket}_{60}$ and $\mathrm{Ket}_{200}$ groups, respectively), which indicated that ketamine downregulated the expression of pro-inflammatory cytokines and mediators, while promoting the expression of anti-inflammatory cytokines.

\section{Discussion}

Current therapeutic strategies for OA are not curative, and are limited to providing symptomatic relief and pain control. Commonly used non-steroidal anti-inflammatory therapeutic agents are associated with adverse gastrointestinal and cardiovascular side-effects. Previous studies regarding the pathogenesis of OA remain inconclusive $(28,29)$, which is hindering the development of effective therapeutic agents for OA. Risk factors for the pathogenesis and progression of OA include age, mechanical injury to the joint, increased expression of metalloproteinases associated with destruction of the cartilage matrix, and impaired repair mechanisms (30-32). Recently, the role of inflammation in the onset and development of OA has been reported in animal and human studies (29). Thus, anti-inflammatory strategies are considered to be an effective approach. In the present study, a rabbit OA model was used to demonstrate the dose-dependent effect of ketamine in ameliorating pathological changes in the knee joint and modulating the expression levels of inflammatory mediators. 
A

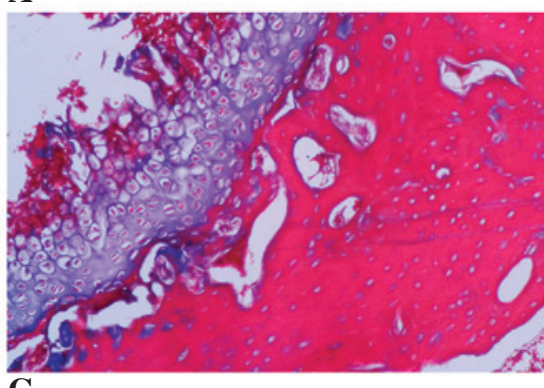

C

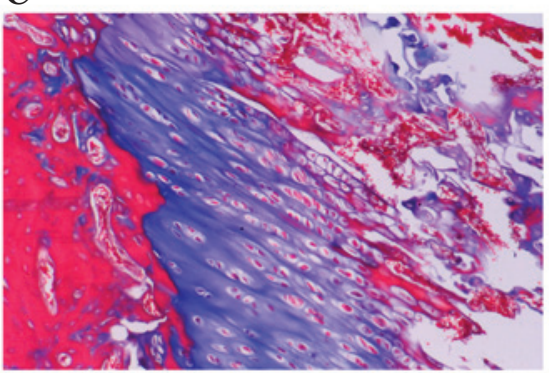

B

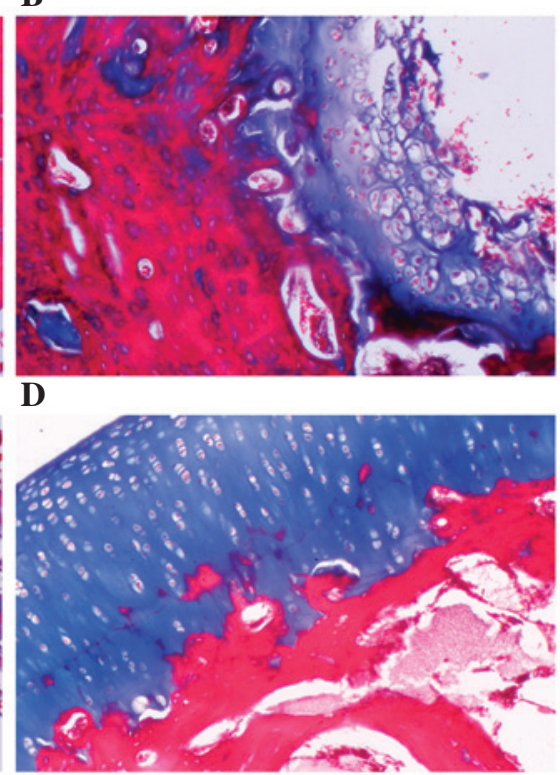

Figure 4. Alcian blue/periodic-acid Schiff staining of articular cartilage samples from osteoarthritis rabbits with or without 60 , 100 and $200 \mu$ mol/1 ketamine. The Alcian blue staining is specific for the cartilaginous tissues while the red periodic-acid Schiff staining indicates the subchondral bone and other tissues. Dose-dependent increases in the thickness of blue-stained cartilaginous tissues subsequent to ketamine treatment were observed: (A) Normal saline; (B) Ket 60 ; (C) $\mathrm{Ket}_{100}$; and (D) $\mathrm{Ket}_{200}$. Magnification x200. The cartilaginous tissues were stained blue and other tissues were stained red.

A well-established rabbit model was produced in the current study by knee immobilization, as previously described (27). Plaster bandages were used to immobilize the knee joint, and OA was induced in the flexed position. The underlying principle was associated with the contracture of the joint and muscle, increase in the load-bearing surface, and changes in biomechanics of the cartilage; leading to cartilage degeneration. Okazaki et al (33) found that articular cartilage degeneration occurred as early as one week after the rabbit knee was immobilized in extension. Furthermore, Kojima et al (34) demonstrated permanent and irreversible changes in cartilage and synovial membrane following four weeks of knee immobilization at full flexion. Compared with other experimental OA models involving therapeutic agent injection or surgical trauma, the knee immobilization model better simulated the natural process of OA pathogenesis. In the present study, knee immobilization for six weeks led to morphological and structural changes in articular cartilage and synovial membrane, along with infiltrating inflammatory cells and elevated levels of pro-inflammatory mediators including IL-1, IL-6, TNF- $\alpha$ and NO, which are typical of OA.

The glutamate/NMDA receptor signaling pathway mediates OA progression. In a rat model of collagenase-induced $\mathrm{OA}$, intra-articular injection of magnesium sulfate, a non-competitive antagonist of the NMDA receptor, inhibited chondrocyte apoptosis and attenuated cartilage degeneration and synovitis (35). In patients undergoing arthroscopic knee surgery, intra-articular magnesium sulfate injection alleviated postoperative pain (36). In addition, previous studies revealed altered NMDA receptor signaling in OA mediated by specific NMDA receptor, NMDA receptor subtype 2B subunit expression in chondrocytes (37). However, clinical applications of NMDA receptor blockers in managing and treating OA is limited by serious side-effects, such as hallucinations,

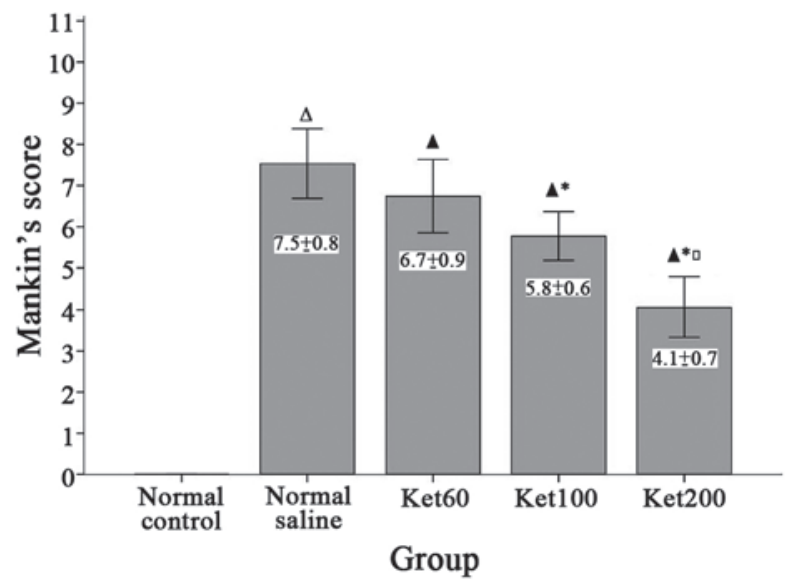

Figure 5. Effect of ketamine on Mankin's scores. Rabbits without any treatment (normal control), or osteoarthritis rabbits treated with normal saline or 60,100 and $200 \mu \mathrm{mol} / 1$ ketamine were analyzed. Histopathological grading of the cartilage was conducted using the Mankin's score system. ${ }^{\wedge} \mathrm{P}<0.01$ vs. the normal control group; ${ }^{\wedge} \mathrm{P}<0.01$ vs. the normal saline group; ${ }^{*} \mathrm{P}<0.01$ vs. the $\mathrm{Ket}_{60}$ group; ${ }^{口} \mathrm{P}<0.01$ vs. the $\mathrm{Ket}_{100}$ group.

memory loss and motor function defects. As an antagonist and allosteric inhibitor of the NMDA receptor, ketamine has been widely administered in clinical practice. In addition to conventional anesthetic and analgesic effects, it manifests anti-inflammatory activity, preserves brain function and relieves bronchial spasm $(21,38)$. Furthermore, intra-articular injection generally requires smaller doses and the plasma concentration of ketamine is particularly low, which greatly reduces the incidence of adverse reactions in the central nervous system (39). Ketamine appears to be a strong candidate for treating OA. Sakai et al (22) revealed that lipopolysaccharide-induced NF- $\mathrm{kB}$ activation was inhibited by pre-treatment with 0.1-1 $\mu \mathrm{mol} / \mathrm{l}$ ketamine in A172 human glioblastoma cells. 
$\mathbf{A}$

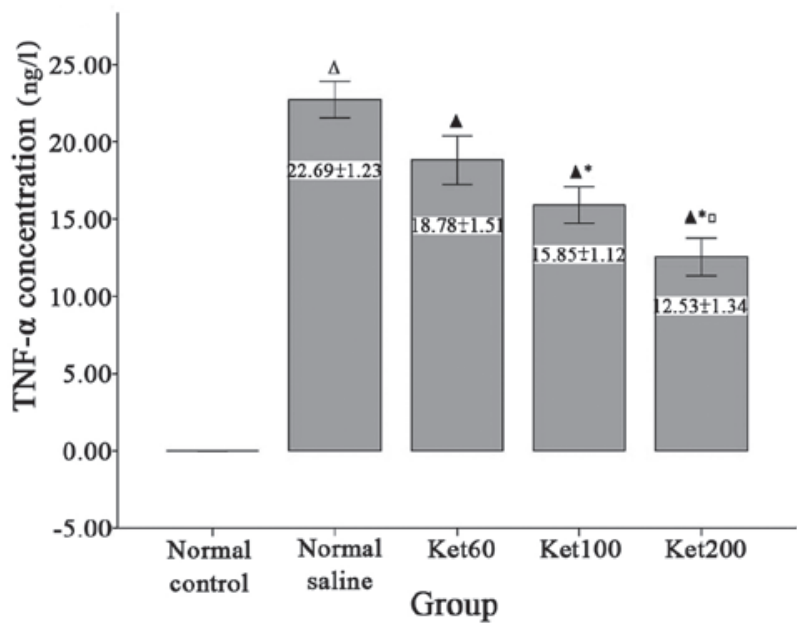

B

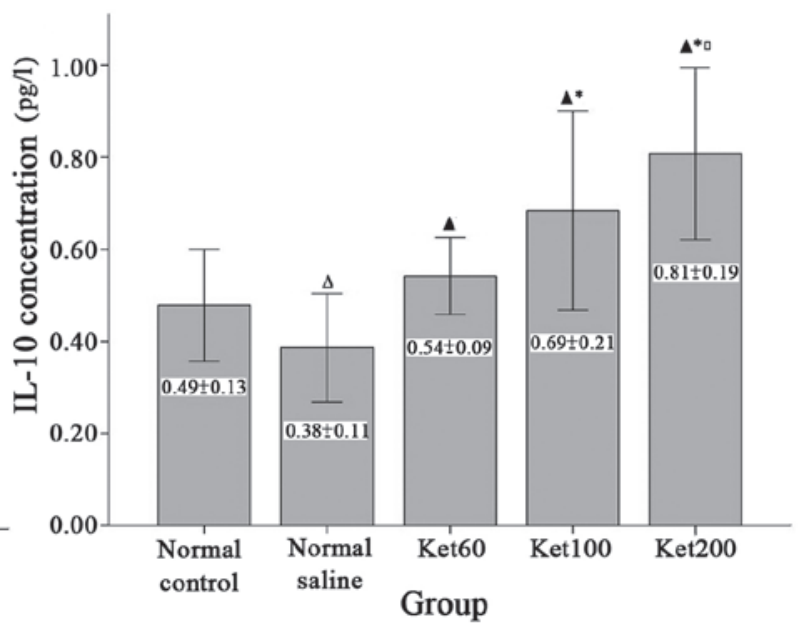

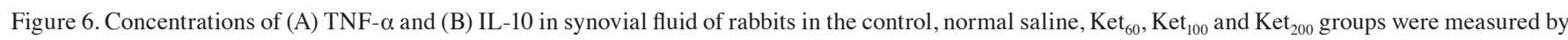
enzyme-linked immunosorbent assay. ${ }^{\wedge} \mathrm{P}<0.01$ vs. the normal control group; ${ }^{\mathbf{}} \mathrm{P}<0.01$ vs. the normal saline group; ${ }^{*} \mathrm{P}<0.01$ vs. the Ket ${ }_{60}$ group; $^{\square} \mathrm{P}<0.01$ vs. the $\mathrm{Ket}_{100}$ group. TNF, tumor necrosis factor; IL, interleukin.

A

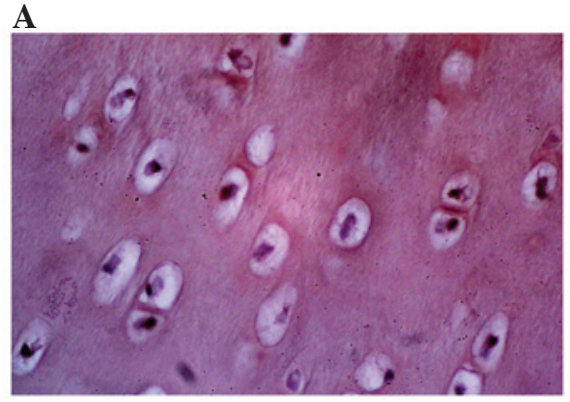

C

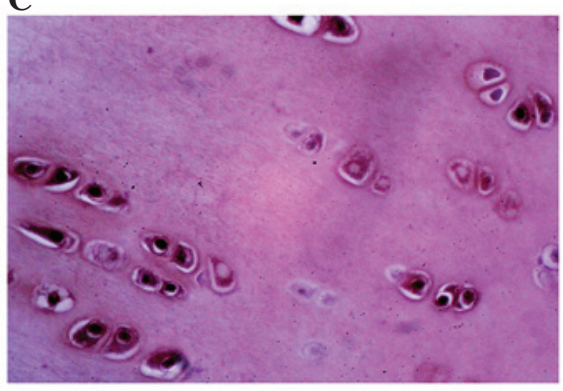

E

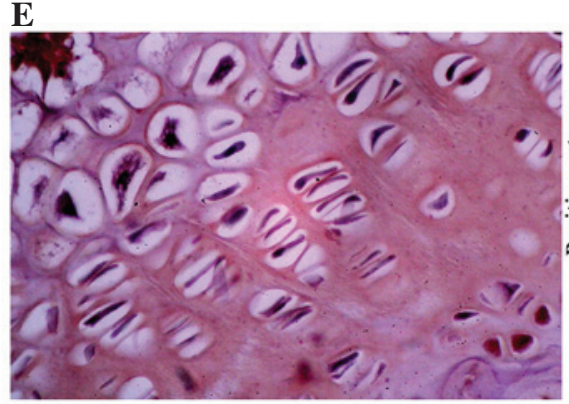

B

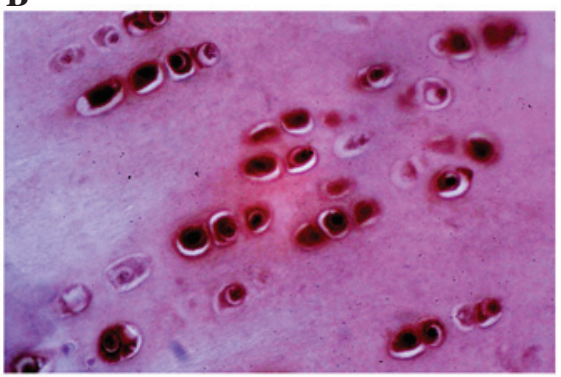

D

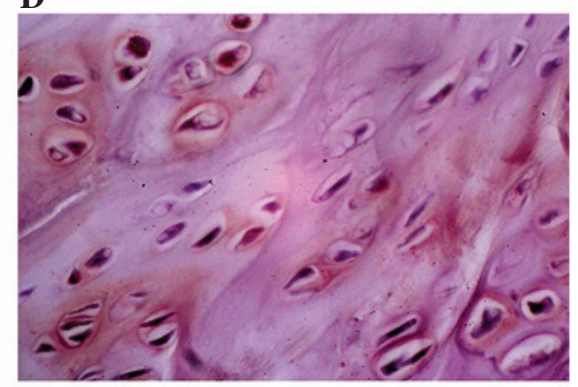

F

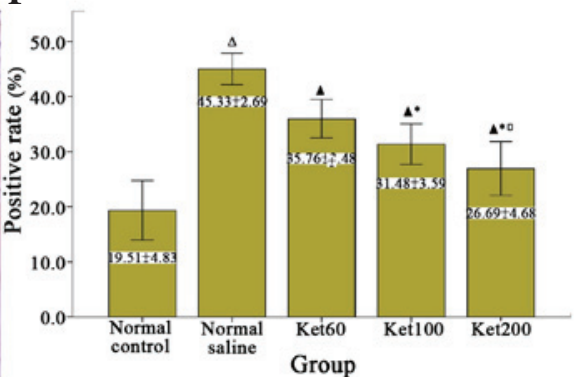

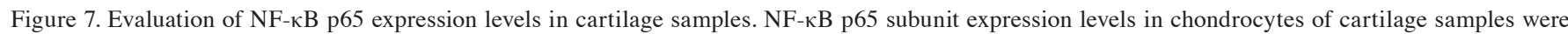
detected by immunohistochemistry with p65-specific antibodies. Representative images of the (A) control, (B) normal saline, (C) Ket ${ }_{60}$, (D) Ket ${ }_{100}$ and (E) Ket 200 groups are presented (magnification, $\mathrm{x} 400)$. (F) Positive rate (\%) of NF- $\kappa \mathrm{B}$ p 65 in each group was calculated from eight randomly selected microscopic fields

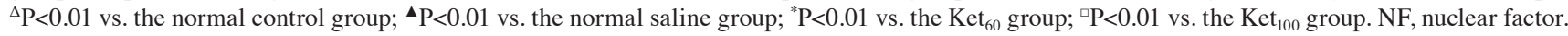

In vivo experiments revealed efficient inhibition of NF- $\mathrm{KB}$ activity with an intravenous ketamine dose of $2.5-10 \mathrm{mg} / \mathrm{kg}$, resulting in a plasma concentration of $60-80 \mu \mathrm{mol} / 1$, which is similar to the dosage used in clinical practice $(40,41)$. To the best of our knowledge, the present study represents the first investigation of ketamine in the potential treatment of $\mathrm{OA}$ in 
a rabbit model, and three different doses of ketamine were investigated. The results indicated that the anti-inflammatory effect of ketamine in OA was dose-dependent, and the highest efficiency was observed at a $200-\mu \mathrm{mol} / 1$ intra-articular dose. Further studies are required to determine the duration of the anti-inflammatory effects of ketamine and assess its long-term effects.

The findings of the current study demonstrated the efficacy of targeting inflammatory mediators in OA pathogenesis. TNF- $\alpha$ has been demonstrated to be essential in cartilage degeneration. Synovitis usually prevails in the early stages of OA, and TNF- $\alpha$ is detected in the majority of synovial fluid samples, indicating that TNF- $\alpha$ is one of the earliest inflammatory cytokines involved in OA onset $(7,42)$. TNF- $\alpha$ activates multinucleated giant cells and stimulates synovial cells to produce prostaglandins and other inflammatory mediators, contributing to matrix degradation, chondrocyte apoptosis and cartilage metabolic disorder; thus, resulting in the pathogenesis and progression of OA (43). In the current study, TNF- $\alpha$ was not detected in the normal control rabbits, but high expression levels of TNF- $\alpha$ were identified in the synovial fluid of OA rabbits. Ketamine effectively reduced the concentration of the inflammatory cytokine, TNF- $\alpha$. Similarly, NF- $\kappa \mathrm{B}$ activation is considered to be important in mediating inflammatory responses associated with OA. Previous studies have evaluated the role of $\mathrm{NF}-\kappa \mathrm{B}$ inhibitors in preventing chondrocyte apoptosis and ameliorating articular cartilage damage; however, the efficacy of these inhibitors has not been extensively investigated in OA animal models (44). The current results revealed an inverse association between ketamine injection and $\mathrm{NF}-\kappa \mathrm{B}$ p65 expression levels in cartilage samples from OA rabbits, demonstrating the potential of ketamine administration to regulate the $\mathrm{NF}-\kappa \mathrm{B}$ signaling pathway and thus preventing OA development.

IL-10 is a major anti-inflammatory cytokine that modulates inflammatory responses in various types of disease, including multiple sclerosis, systemic lupus erythematosus and inflammatory bowel disease $(45,46)$. Furthermore, IL-10 directly inhibits the production of pro-inflammatory cytokines (47), including TNF- $\alpha$ in the joint cavity in multiple experimental models of arthritic animals $(48,49)$. In the OA rabbit model of the present study, IL-10 expression levels in the synovial fluid were significantly reduced; however, upon ketamine treatment, the IL-10 levels increased in a dose-dependent manner. The underlying molecular mechanism, however, remains unknown and further investigation is required.

In conclusion, the pathogenesis of OA is a particularly complicated process, which is mediated by chondrocyte apoptosis, matrix destruction, cartilage degeneration, metabolic disorder and altered signal transduction. The results of the current study provide support for anti-inflammatory therapeutic strategies, using ketamine, for the treatment of OA. However, additional investigations are required to establish the optimal therapeutic dosage of ketamine in OA, and to elucidate the molecular mechanisms underlying the regulation of inflammation. Controlled multicenter clinical trials are required to provide an evidence-based therapeutic strategy for possible clinical application.

\section{Acknowledgements}

The present study was supported by the Science and Technology Foundation of Guizhou Province, China [grant no. (2011)2244].

\section{References}

1. Felson DT, Naimark A, Anderson J, Kazis L, Castelli W and Meenan RF: The prevalence of knee osteoarthritis in the elderly. The framingham osteoarthritis study. Arthritis Rheum 30: 914-918, 1987.

2. Zhang Y, Xu L, Nevitt MC, Aliabadi P, Yu W, Qin M, Lui LY and Felson DT: Comparison of the prevalence of knee osteoarthritis between the elderly Chinese population in Beijing and whites in the United States: The Beijing osteoarthritis study. Arthritis Rheum 44: 2065-2071, 2001.

3. Kuettner KE and Cole AA: Cartilage degeneration in different human joints. Osteoarthritis Cartilage 13: 93-103, 2005.

4. Pelletier JP, Martel-Pelletier J and Abramson SB: Osteoarthritis, an inflammatory disease: Potential implication for the selection of new therapeutic targets. Arthritis Rheum 44: 1237-1247, 2001.

5. Abramson SB, Attur M, Amin AR and Clancy R: Nitric oxide and inflammatory mediators in the perpetuation of osteoarthritis. Curr Rheumatol Rep 3: 535-541, 2001.

6. Mcinnes IB and Liew FY: Cytokine networks-towards new therapies for rheumatoid arthritis. Nat Clin Pract Rheumatol 1: 31-39, 2005

7. Smith MD, Triantafillou S, Parker A, Youssef PP and Coleman M: Synovial membrane inflammation and cytokine production in patients with early osteoarthritis. J Rheumatol 24: 365-371, 1997.

8. Dougados M, Combe B, Braun J, Landewé R, Sibilia J, Cantagrel A, Feydy A, Van Der Heijde D, Leblanc V and Logeart I: A randomised, multicentre, double-blind, placebo-controlled trial of etanercept in adults with refractory heel enthesitis in spondyloarthritis: The HEEL trial. Ann Rheum Dis 69: 1430-1435, 2010.

9. Fioravanti A, Fabbroni M, Cerase A and Galeazzi M: Treatment of erosive osteoarthritis of the hands by intra-articular infliximab injections: A pilot study. Rheumatol Int 29: 961-965, 2009.

10. Louthrenoo W, Nilganuwong S, Aksaranugraha S, Asavatanabodee P and Saengnipanthkul S; Thai Study Group: The efficacy, safety and carry-over effect of diacerein in the treatment of painful knee osteoarthritis: A randomised, double-blind, NSAID-controlled study. Osteoarthritis Cartilage 15: 605-614, 2007.

11. Skerry TM and Genever PG: Glutamate signalling in non-neuronal tissues. Trends Pharmacol Sci 22: 174-181, 2001.

12. Hinoi E, Takarada T, Ueshima T, Tsuchihashi Y and Yoneda Y: Glutamate signaling in peripheral tissues. Eur J Biochem 271: $1-13,2004$.

13. Kalariti N, Pissimissis N and Koutsilieris M: The glutamatergic system outside the CNS and in cancer biology. Expert Opin Investig Drugs 14: 1487-1496, 2005.

14. Mcnearney T, Baethge BA, Cao S, Alam R, Lisse JR and Westlund KN: Excitatory amino acids, TNF-alpha, and chemokine levels in synovial fluids of patients with active arthropathies. Clin Exp Immunol 137: 621-627, 2004.

15. Laube B, Hirai H, Sturgess M, Betz H and Kuhse J: Molecular determinants of agonist discrimination by NMDA receptor subunits: Analysis of the glutamate binding site on the NR2B subunit. Neuron 18: 493-503, 1997.

16. Flood S, Parri R, Williams A, Duance V and Mason D: Modulation of interleukin-6 and matrix metalloproteinase 2 expression in human fibroblast-like synoviocytes by functional ionotropic glutamate receptors. Arthritis Rheum 56: 2523-2534, 2007.

17. Lawand NB, Willis WD and Westlund KN: Excitatory amino acid receptor involvement in peripheral nociceptive transmission in rats. Eur J Pharmacol 324: 169-177, 1997.

18. Bondok RS and Abd El-Hady AM: Intra-articular magnesium is effective for postoperative analgesia in arthroscopic knee surgery. Br J Anaesth 97: 389-392, 2006.

19. Lee CH, Wen ZH, Chang YC, Huang SY, Tang CC, Chen WF, Hsieh SP, Hsieh CS and Jean YH: Intra-articular magnesium sulfate $(\mathrm{MgSO} 4)$ reduces experimental osteoarthritis and nociception: Association with attenuation of N-methyl-D-aspartate (NMDA) receptor subunit 1 phosphorylation and apoptosis in rat chondrocytes. Osteoarthritis Cartilage 17: 1485-1493, 2009. 
20. Irifune $M$, Shimizu $T$, Nomoto $M$ and Fukuda $T$ : Ketamine-induced anesthesia involves the N-methyl-D-aspartate receptor-channel complex in mice. Brain Res 596: 1-9, 1992.

21. Loix S, De Kock M and Henin P: The anti-inflammatory effects of ketamine: State of the art. Acta Anaesthesiol Belg 62: 47-58, 2011.

22. Sakai T, Ichiyama T, Whitten CW, Giesecke AH and Lipton JM: Ketamine suppresses endotoxin-induced NF-kappaB expression. Can J Anaesth 47: 1019-1024, 2000.

23. Gokcinar D, Ergin V, Cumaoglu A, Menevse A and Aricioglu A: Effects of ketamine, propofol, and ketofol on proinflammatory cytokines and markers of oxidative stress in a rat model of endotoxemia-induced acute lung injury. Acta Biochim Pol 60: 451-456, 2013.

24. Videman T: Experimental osteoarthritis in the rabbit: Comparison of different periods of repeated immobilization. Acta Orthop Scand 53: 339-347, 1982.

25. Pelletier JP, Jovanovic D, Fernandes JC, Manning P, Connor JR Currie MG, Di Battista JA and Martel-Pelletier J: Reduced progression of experimental osteoarthritis in vivo by selective inhibition of inducible nitric oxide synthase. Arthritis Rheum 41: 1275-1286, 1998.

26. Mankin HJ, Dorfman H, Lippiello L and Zarins A: Biochemical and metabolic abnormalities in articular cartilage from osteo-arthritic human hips. II. Correlation of morphology with biochemical and metabolic data. J Bone Joint Surg Am 53: 523-537, 1971.

27. Langenskiöld A, Michelsson JE and Videman T: Osteoarthritis of the knee in the rabbit produced by immobilization. Attempts to achieve a reproducible model for studies on pathogenesis and therapy. Acta Orthop Scand 50: 1-14, 1979.

28. Felson DT: Developments in the clinical understanding of osteoarthritis. Arthritis Res Ther 11: 203, 2009.

29. Sokolove J and Lepus CM: Role of inflammation in the pathogenesis of osteoarthritis: Latest findings and interpretations. Ther Adv Musculoskelet Dis 5: 77-94, 2013.

30. Goldring MB and Goldring SR: Articular cartilage and subchondral bone in the pathogenesis of osteoarthritis. Ann N Y Acad Sci 1192: 230-237, 2010.

31. Woessner JF Jr and Gunja-Smith Z: Role of metalloproteinases in human osteoarthritis. J Rheumatol Suppl 27: 99-101, 1991.

32. Blaney Davidson EN, Scharstuhl A, Vitters EL, Van Der Kraan PM and Van Den Berg WB: Reduced transforming growth factor-beta signaling in cartilage of old mice: Role in impaired repair capacity. Arthritis Res Ther 7: R1338-R1347, 2005.

33. Okazaki R, Sakai A, Otsuyama A, Sakata T, Nakamura T and Norimura T: Apoptosis and p53 expression in chondrocytes relate to degeneration in articular cartilage of immobilized knee joints. J Rheumatol 30: 559-566, 2003.

34. Kojima S, Hoso M, Watanabe M, Matsuzaki T, Hibino I and Sasaki K: Experimental joint immobilization and remobilization in the rats. J Phys Ther Sci 26: 865-871, 2014.

35. Lee CH, Wen ZH, Chang YC, Huang SY, Tang CC, Chen WF, Hsieh SP, Hsieh CS and Jean YH: Intra-articular magnesium sulfate $(\mathrm{MgSO} 4)$ reduces experimental osteoarthritis and nociception: Association with attenuation of N-methyl-D-aspartate (NMDA) receptor subunit 1 phosphorylation and apoptosis in rat chondrocytes. Osteoarthritis Cartilage 17: 1485-1493, 2009.
36. Bondok RS and Abd El-Hady AM: Intra-articular magnesium is effective for postoperative analgesia in arthroscopic knee surgery. Br J Anaesth 97: 389-392, 2006.

37. Ramage L, Martel MA, Hardingham GE and Salter DM: NMDA receptor expression and activity in osteoarthritic human articular chondrocytes. Osteoarthritis Cartilage 16: 1576-1584, 2008.

38. Agrawal A and Shrivastava J: Intravenous ketamine for refractory bronchospasm precipitated by $\mathrm{H} 1 \mathrm{~N} 1$ infection. Front Pediatr 2: 24, 2014.

39. Akhondzade R, Pipelzade MR, Gousheh MR, Sarrafan N and Mahmoodi K: Comparison of the analgesic effect of intraarticular and extra-articular injection of morphine and ketamine compound in arthrotomy lower limb surgery under spinal anesthesia. Pak J Med Sci 30: 942-945, 2014.

40. Idvall J, Ahlgren I, Aronsen KR and Stenberg P: Ketamine infusions: Pharmacokinetics and clinical effects. Br J Anaesth 51: 1167-1173, 1979.

41. Wieber J, Gugler R, Hengstmann JH and Dengler HJ: Pharmacokinetics of ketamine in man. Anaesthesist 24: 260-263, 1975.

42. Venn G, Nietfeld JJ, Duits AJ, Brennan FM, Arner E, Covington M, Billingham ME and Hardingham TE: Elevated synovial fluid levels of interleukin-6 and tumor necrosis factor associated with early experimental canine osteoarthritis. Arthritis Rheum 36: 819-826, 1993.

43. Botha-Scheepers S, Watt I, Slagboom E, De Craen AJ, Meulenbelt I, Rosendaal FR, Breedveld FC, Huizinga TW and Kloppenburg M: Innate production of tumour necrosis factor alpha and interleukin 10 is associated with radiological progression of knee osteoarthritis. Ann Rheum Dis 67: 1165-1169, 2008.

44. Saklatvala J: Inflammatory signaling in cartilage: MAPK and NF-kappaB pathways in chondrocytes and the use of inhibitors for research into pathogenesis and therapy of osteoarthritis. Curr Drug Targets 8: 305-313, 2007.

45. Beebe AM, Cua DJ and de Waal Malefyt R: The role of interleukin-10 in autoimmune disease: Systemic lupus erythematosus (SLE) and multiple sclerosis (MS). Cytokine Growth Factor Rev 13: 403-412, 2002.

46. Tilg H, van Montfrans C, van den Ende A, Kaser A, van Deventer SJ, Schreiber S, Gregor M, Ludwiczek O, Rutgeerts P, Gasche C, et al: Treatment of Crohn's disease with recombinant human interleukin 10 induces the proinflammatory cytokine interferon gamma. Gut 50: 191-195, 2002.

47. de Waal Malefyt R, Abrams J, Bennett B, Figdor CG and De Vries JE: Interleukin 10(IL-10) inhibits cytokine synthesis by human monocytes: An autoregulatory role of IL-10 produced by monocytes. J Exp Med 174: 1209-1220, 1991.

48. Lubberts E, Joosten LA, Van Den Bersselaar L, Helsen MM, Bakker AC, Xing Z, Richards CD and Van Den Berg WB: Intra-articular IL-10 gene transfer regulates the expression of collagen-induced arthritis (CIA) in the knee and ipsilateral paw. Clin Exp Immunol 120: 375-383, 2000.

49. Lechman ER, Jaffurs D, Ghivizzani SC, Gambotto A, Kovesdi I, Mi Z, Evans CH and Robbins PD: Direct adenoviral gene transfer of viral IL-10 to rabbit knees with experimental arthritis ameliorates disease in both injected and contralateral control knees. J Immunol 163: 2202-2208, 1999. 